\title{
Part-task simulation of synthetic and enhanced vision concepts for lunar landing
}

\author{
Jarvis (Trey) J. Arthur, III ${ }^{a}$, Randall E. Bailey ${ }^{a}$, E. Bruce Jackson ${ }^{a}$, James R. Barnes ${ }^{b}$, \\ Steven P. Williams ${ }^{a}$ and Lynda J. Kramer ${ }^{a}$ \\ ${ }^{a}$ NASA Langley Research Center, Hampton, VA \\ ${ }^{b}$ ARINC Inc, Hampton, VA
}

\begin{abstract}
During Apollo, the constraints placed by the design of the Lunar Module (LM) window for crew visibility and landing trajectory were "a major problem." Lunar landing trajectories were tailored to provide crew visibility using nearly 70 degrees look-down angle from the canted LM windows. Apollo landings were scheduled only at specific times and locations to provide optimal sunlight on the landing site.

The complications of trajectory design and crew visibility are still a problem today. Practical vehicle designs for lunar lander missions using optimal or near-optimal fuel trajectories render the natural vision of the crew from windows inadequate for the approach and landing task. Further, the sun angles for the desirable landing areas in the lunar polar regions create visually powerful, season-long shadow effects. Fortunately, Synthetic and Enhanced Vision (S/EV) technologies, conceived and developed in the aviation domain, may provide solutions to this visibility problem and enable additional benefits for safer, more efficient lunar operations. Piloted simulation evaluations have been conducted to assess the handling qualities of the various lunar landing concepts, including the influence of cockpit displays and the informational data and formats. Evaluation pilots flew various landing scenarios with S/EV displays. For some of the evaluation trials, an eye glasses-mounted, monochrome monocular display, coupled with head tracking, was worn. The head-worn display scene consisted of S/EV fusion concepts.

The results of this experiment showed that a head-worn system did not increase the pilot's workload when compared to using just the head-down displays. As expected, the head-worn system did not provide an increase in performance measures. Some pilots commented that the head-worn system provided greater situational awareness compared to just head-down displays.
\end{abstract}

Keywords: Head-worn display, Synthetic Vision, Enhanced Vision, Cockpit, Moon, Spacecraft, Simulation

\section{INTRODUCTION}

As NASA embarked on the development of the Constellation Program platforms, a fundamental understanding of spacecraft handling qualities is paramount to successful design. A research, development, test, and evaluation process approach was used to identify, understand, and interpret the engineering and human factors principles which govern the pilot-vehicle dynamic system as they pertain to space exploration missions and tasks.

Toward this objective, the results of a piloted simulation are described. ${ }^{1}$ This work investigated the handling qualities of a next generation Lunar Landing vehicle and the influence of critical design issues that may make significant impacts on the design and its operational employment. It is important in this context to recall that handling qualities include much more than just stability and control characteristics of a spacecraft or aircraft. Handling qualities are affected by all aspects of the "pilot-vehicle dynamic system," including the motion, visual and aural cues of the vehicle response as the pilot performs the required operation or task. This paper presents the results of a piloted simulation evaluation of control and display interactions on the handling qualities of a lunar landing vehicle.

The use of a head-tracked Head-Worn Display (HWD) was also investigated in conjunction with the Lunar Lander handling qualities experiment. NASA has conducted aviation-specific research using HWD and demonstrated its potential as a full field of regard display. ${ }^{2}$

\footnotetext{
Further author information: Trey Arthur, E-mail: Trey.Arthur@nasa.gov, Telephone: 1-757-864-6609
} 


\section{BACKGROUND}

NASA's Vision for Space Exploration under the Constellation program included the return of manned missions to the lunar surface. While autonomous capabilities were anticipated, manual piloted control of the lunar landing would be critical especially where "the pilot's ability to perceive the state of his vehicle and to apply alternatives to the nominal control is an asset." ${ }^{3}$ The Apollo design highlights the challenges for the lunar landing mission.

Though there is no weather on the moon, impaired vision for the flight crew can still create technical and operational challenges or problems for lunar or planetary landings. Thrusters can create a dust cloud that can significantly reduce visibility during the critical landing phase of the flight. During Apollo, the final $100 \mathrm{ft}$ to landing was generally obscured by blowing dust, creating "a little trouble figuring out cross-range and downrange velocities." 4 The absence of atmospheric haze in the lunar environment makes shadows significantly more stark, and increases the propensity for visual illusions, or removal of critical visual cues while in shadowed conditions. Landing at polar sites, in the presence of significant terrain, or in darkness, will exacerbate this problem. In addition, the attitude of the spacecraft, coupled with the window location and size, directly affects the out-the-window visibility for the crew. Unlike atmospheric aircraft that generally point in the direction they are going, spacecraft are comparatively unconstrained in attitude. Thus, a spacecraft can have its windows facing $180^{\circ}$ from the direction of travel. If the vehicle trajectory can be optimized without crew visibility being a constraint, significant fuel and weight savings may be gained. During Apollo, crew visibility and trajectory (fuel) were traded off in the design. "The constraints placed on crew visibility by the design of the LM (Lunar Module) window and by the trajectory parameters make the viewing of the programmed landing site a major problem." 5

The use of Synthetic Vision (SV) and Enhanced Vision (EV) may obviate some of the constraints and penalties placed on the Apollo designs. Synthetic Vision System (SVS) may provide three key benefits for lunar and planetary applications. ${ }^{6}$ First, the designer controls the SVS scene lighting, terrain coloring and virtual camera angles. Therefore, visual cues for the landing site can be created in the SVS independent of the sunangle. Second, the information is "full field-of-regard." SV terrain presentations may be provided, conformally, in any location with respect to the vehicle or its actual window placement as required to successfully accomplish the mission. Lastly, SV provides the capability for "augmented reality" - that is, the enhancement of existing visual cues or provide conformal overlay of important vehicle state information such as forward and down velocities, altitude, and fuel remaining can be overlaid directly onto the terrain display to significantly ease pilot interpretation of the data and enhance situation awareness.

EV, a real-time imaging sensor, can provide two key benefits for this task. First, in lieu of a high resolution lunar terrain database, active sensors could be used to generate this same information. More likely, however, onboard sensors will be used to supplement the SVS display in real-time, for instance, to ensure the landing site is free of objects not in the database (such as cultural artifacts on a colonized Moon). A significant aviation-domain background in research and flight experimentation/experience for optimal fusion of synthetic and enhanced vision (such as Forward Looking Infrared (FLIR)) is being used as reference for this work. ${ }^{7,8}$ Active sensors should be capable of detecting items which are not stored in a static, onboard database. Further, active sensors have been developed and tested to provide additional or secondary sources for navigation, independent of the aircraft's primary navigation sources. These sensors may serve as independent integrity checks to the primary sensors or as a primary means for navigation position information when other sources are not available. Research, development, test and evaluation was being used to define, from a flight deck-centric view, SV and EV roles, technologies, methods, and design trade-spaces such as field-of-view, field-of-regard, update rate, and other display parameters as they impact the Constellation Lunar Lander Vehicle.

Coupling a head-tracked HWD with Synthetic and Enhanced Vision (S/EV) concepts has shown encouraging results in the aviation domain. ${ }^{2}$ Pilots prefer to have cockpit information on a head-up ${ }^{9}$ display so they do not have to look head-down for critical flight information. Further, a Head-Up Display (HUD) or HWD allows pilots to continue their scan patterns out the window while having flight instrument information easily in view. However, HWDs have some advantages compared to a HUD. For example, a HWD has an unlimited field-ofregard while the HUD viewing volume is fixed and constrained by size of the combiner glass. Given that a spacecraft is unconstrained in attitude, a fixed, forward facing HUD may not be ideal. Another advantage over a HUD, perhaps the most important, is the overall weight of an HWD system. For the Apollo Lunar Module (LM), 
every $1 \mathrm{~kg}$ of non-propellant weight resulted in adding over $3 \mathrm{~kg}$ of LM fuel. And by adding $4 \mathrm{~kg}$ of LM payload, $50 \mathrm{~kg}$ of Saturn V propellant had to be added. ${ }^{10}$ Weight was so critical to the LM design, a team was formed to find ways to reduce the LM weight. ${ }^{10}$ In fact, several iterations of the weight reduction called (unsuccessfully) for the removal of the video camera that recorded the first step on the moon.

\section{EXPERIMENTAL EVALUATION}

A pilot-in-the-loop simulation was conducted to explore the control and display interactions on the handling qualities of a lunar lander vehicle. Varied in the experiment was the rotational control power (amount of maneuverability), the guidance symbology, glideslope trajectory and the use of a HWD. The results presented in this paper focus on the influence of the HWD. This work builds from the previous work ${ }^{11,12}$ in this area as control and display interactions strongly impact several design considerations in a lunar lander design.

The critical influence of visual cues was emphasized in this experiment. As the trajectory approach angle is changed, the pitch attitude will vary, affecting the amount of time that the crew can use natural vision viewing of the landing site. The previous work ${ }^{11,12}$ showed that manual flight to an offset landing zone was not possible. One might consider that this result was because the out-the-window simulated visual cues were insufficient to support the task (as discussed later). On the other hand, the visual cues might have been sufficient but the control characteristics were deficient. In any event, the influence of natural vision and the potential of "equivalent vision" technologies warranted exploration. Equivalent vision may represent a new tool in the manned planetary exploration designers "tool-bag."

These experimental objectives were evaluated in the context of variations in the control authority of a lunar landing vehicle which uses a rate command-attitude hold $(\mathrm{RCAH})$ control law, analogous to the criteria developed by Cheatham and Hackler. ${ }^{5}$

\subsection{Simulated spacecraft}

The Constellation Program's lunar lander (Altair) was an evolving design attempting to meet many competing design and operational objectives. ${ }^{13}$ The simulated spacecraft was not intended to be a replica of the Altair vehicle, but was roughly representative of its current instantiation to develop relevant design data and guidance material.

A simulation model was built based on the Altair vehicle Design and Analysis Cycle (DAC)-2 configuration. This model roughly approximates vehicles also being used under other projects. ${ }^{14,15}$

The simulation model was built using object-oriented programming techniques within the Langley Standard Realtime Simulation in $\mathrm{C}++(\mathrm{LaSRS}++)$ software framework. ${ }^{16}$ A generic-spacecraft simulation within this framework served as a test bed for modeling spacecraft dynamics, propulsion, control systems, guidance, and displays. The vehicle model was composed of interconnected stages, simulated using a parent/child relationship. The ascent stage was modeled as the parent with the descent stage as a child. The descent stage contained separate mass models for the main engine fuel, Reaction Control System (RCS) fuel, and the airlock. Force models were provided for the main engine, each of the sixteen RCS engines, and the four landing gear models. For simplicity, the throttle-able, thrust output of the main engine force model (18,647 lbf maximum) was placed close to the lateral and longitudinal axes of the stacked center-of-mass $(\mathrm{cm})$. Active gimbaling of the main engine was provided to trim the thrust vector through the $\mathrm{cm}$. All forces and moments were applied to the stacked vehicles $\mathrm{cm}$.

The mass and principle products of inertia were reduced by approximately $5 \%$ as fuel was expended during this approach and landing task. Small cross-products of inertia were also simulated based upon the simulated vehicle mass distribution. The pilot, as defined by the design eye point reference (DERP), was located $28.1 \mathrm{ft}$ above the extended landing gear position. The center of mass at the initial starting condition was $6.6 \mathrm{ft}$ below the DERP. The four RCS jets were placed in a ring around the descent stage using an orthogonal quadrant of four individual thrusters for attitude control. The RCS thruster size was experimentally varied. The RCS jets were located at a $14.8 \mathrm{ft}$ radial from the centerline of the vehicle, approximately $7.7 \mathrm{ft}$ below the DERP. 

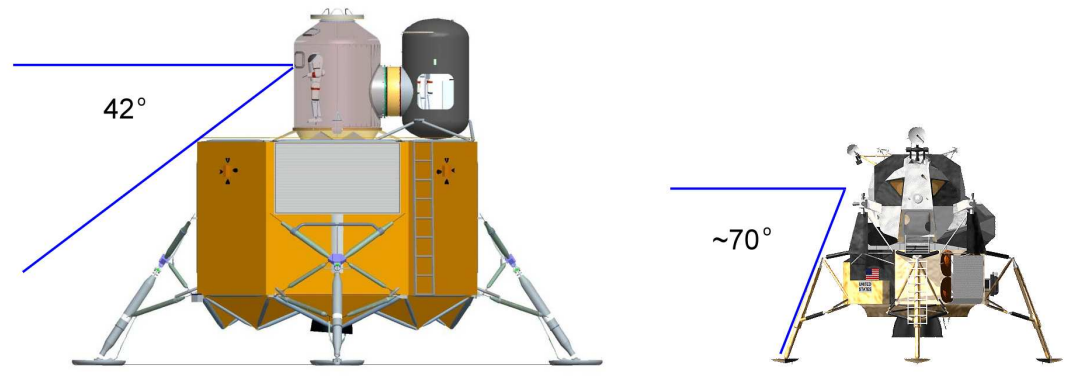

Figure 1. Simulated Lunar Lander-Type Vehicle compared to the Apollo Lunar Lander.

\subsection{Simulator}

The experiment was conducted in the Lunar Flight Deck (LFD) simulation facility at NASA Langley Research Center (LaRC). The Evaluation Pilot (EP) conducted the task in a standing position, analogous to the Apollo Lunar Module design. The crew station is shown in Figure 2. A window (20 inch H x 14 inch V) approximated the field-of-view anticipated for the Lunar Lander vehicle. Two head-down (13.25 inch $\mathrm{H} \mathrm{x} 10.5$ inch V) displays, using 1280 x 1024 pixel resolution, provided primary flight, navigation, and systems awareness for the EP. The EPs used an Apollo-vintage Rotational Hand Controller (RHC) and Translational Hand Controller (THC). The controllers were mechanical and their characteristics were fixed. The left-hand THC was provisioned for 3-axis translational control commands but only up/down vertical commands were active. The right-hand RHC provided 3 -axis rotational control commands, using a base pivot for roll, palm-pivot for pitch, and twist-pivot for yaw.

The LFD used multiple projectors and a dome screen to provide a $135^{\circ}$ horizontal $(\mathrm{H})$ x $67.5^{\circ}$ vertical (V) field-of-view. The viewing surface of the screen was located on a $10.6 \mathrm{ft}$ radius from the DERP. The dome and projection system were biased to provide $22.5^{\circ}$ up and $45.0^{\circ}$ down angle viewing from the DERP. The multiple image generators and projectors were warped and blended to uniformly provide $\sim 30$ pixels per degree resolution across the field-of-view, referenced to the DERP.

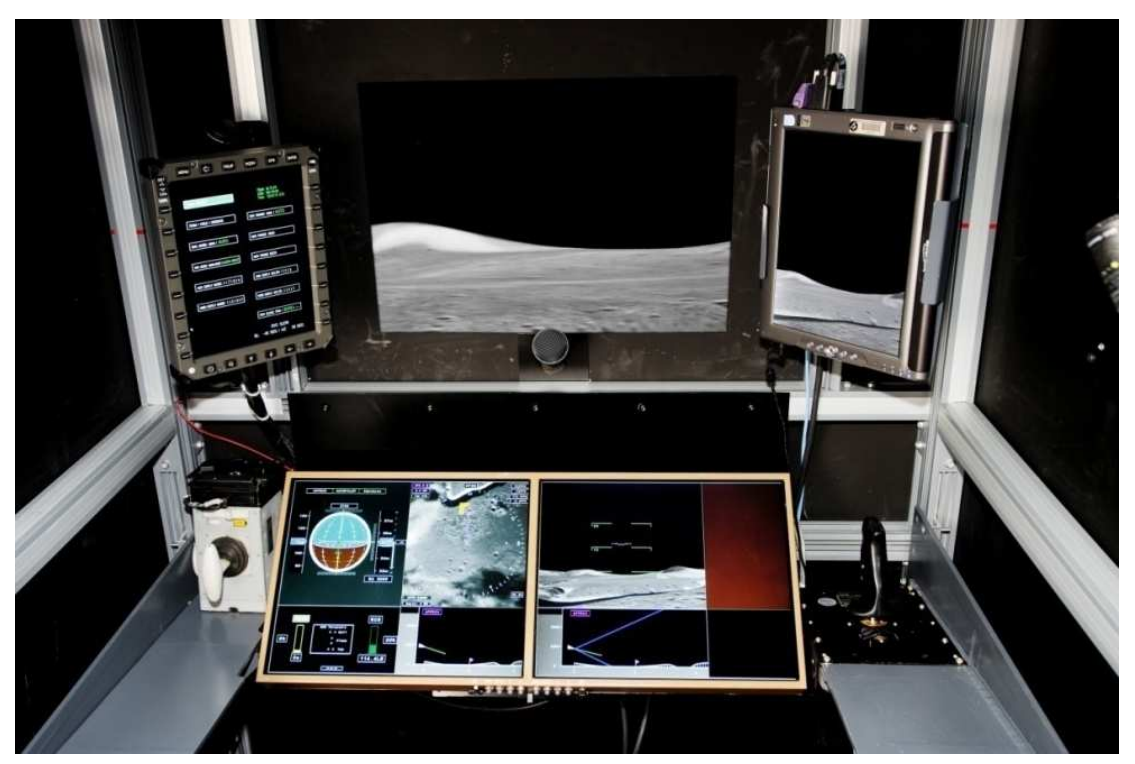

Figure 2. Evaluation Pilot Crew Station. 
A lunar polar landing site was preferred for the evaluations but terrain data of reasonable resolution was not available. Instead, the Apollo 15 landing site was utilized because of the availability of higher resolution data and the interesting terrain features it provided. The Out-the-Window (OTW) lunar database was created using lunar topology and imagery data collected by the Clementine project. ${ }^{17} \mathrm{~A}$ spherical lunar database was created that covered the entire lunar surface and was built with three topology and imagery resolutions. The entire moon was created using a resolution of approximately 4 kilometers per elevation post, draped with 1 kilometer per pixel imagery. The medium resolution used around the area of the Apollo 15 landing site was approximately 500 meters per elevation post, draped with 125 meter per pixel imagery. The highest resolution data was used in the area immediately surrounding the Apollo 15 landing site. This resolution was 15 meters per elevation post-spacing, draped with 4 meter per pixel imagery.

In the landing area, the resolution of the OTW database was good, but not to levels approaching the realworld. The OTW presentation was augmented with the addition of boulder hazards and habitat models to increase the visual density around the landing site. Fifty boulders were pseudo-randomly placed around the Apollo 15 landing site in a 0.015 degree by 0.015 degree area. The boulders ranged from 0.5 meters in diameter to 10 meters in diameter. Various models were created and located around the planned landing site. The models included Lunar Landers, habitats, solar array panels and rover vehicles.

Aural call-outs of the altitude above the terrain (extended gear height) were provided from $1000 \mathrm{ft}$ down to touchdown. The landing call-outs in $10 \mathrm{ft}$ increments started at $150 \mathrm{ft}$ and continued until touchdown. The navigation solution generated by the spacecraft simulation contained no simulated system errors.

\subsection{Evaluation pilots}

Eight EPs flew this experiment, completed in 2009. For 5 of the 8 pilots, some of the data runs were conducted while the EP was wearing a HWD. For most runs, the HWD was not worn and piloting was done with the head down displays and OTW scene. All EPs were graduates of the Air Force Test Pilot School or Naval Test Pilot School and were experienced in aircraft handling qualities evaluations. None were experienced in the actual lunar landing mission. Some had considerable experience in rotary wing vehicles. Two of the EPs were current or former Astronauts.

\subsection{Experiment protocol}

Each EP was given an initial briefing before the start of the experiment, outlining the experimental objectives and an overview of the simulation and the test procedures. At least one hour of training in the simulator was conducted to ensure familiarity with the tasks, the displays, the controllers, the guidance concepts, and the subjective data measures. Data collection followed thereafter. Data collection was nominally limited to 1 hour periods, with 15 minute breaks between, to mitigate fatigue.

For each configuration, the EPs first flew one approach and landing for practice/familiarity, followed by a minimum of two runs for "data." The EP had the option for a third data run if they felt that the two runs exhibited very different characteristics that potentially hampered their handling qualities assessment. Therefore, each configuration consisted of at least 3 (and up to 4) data runs. In order to reduce the amount of donning and removing the HWD, data runs with the HWD were grouped into blocks. A HWD block consisted of 4 configurations, thus a total of at least 12 data runs. EPs donned the HWD during the entire data block which lasted approximately 45 minutes.

Following the tasks, the EPs assigned a pilot rating using the Cooper-Harper rating scale. ${ }^{18}$ Pilot comments were then given, generally prompted via a comment card. Finally, NASA Task Load Index (TLX) workload ratings ${ }^{19}$ were given.

Engineering unit and video data was recorded on all runs. Pilot audio comments were digitally recorded and transcribed. 

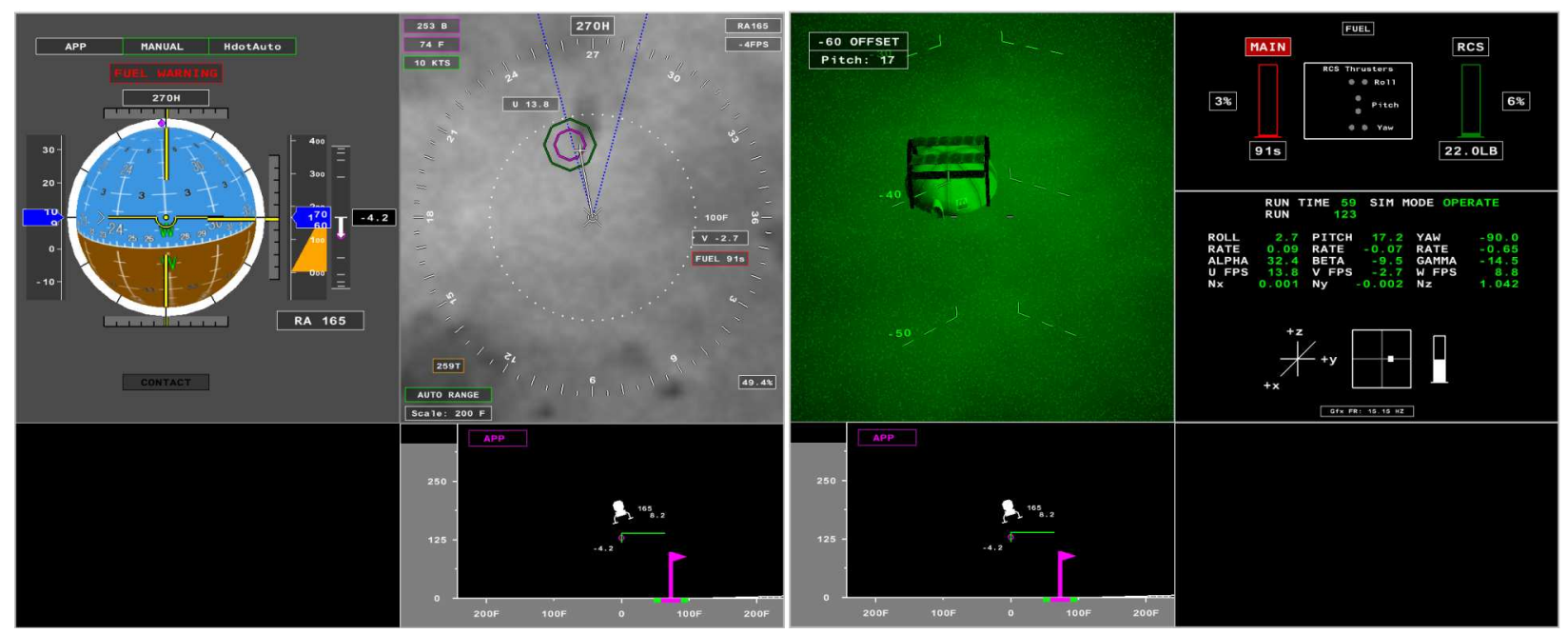

Figure 3. Primary Flight, Navigation, Vertical Situation and Auxiliary Displays.

\subsection{Head-down displays}

Two color head-down displays (HDDs) (see Figure 3) were installed on the forward instrument panel. The two displays showed four primary display formats: 1) Attitude Direction Indicator (ADI); 2) Navigational Display (ND); 3) Vertical Situation Display (VSD); and 4) Auxiliary Display.

The ADI (see Figure 3) served as the primary flight display. The ADI showed lunar attitude reference using a roll/pitch/yaw "eight-ball." The ADI included digital readouts of ground speed (in knots), altitude (in ft, height above the landing zone), radar altitude (in ft, height terrain below extended gear position), and vertical descent rate (in ft/sec). On the vertical descent rate display, a magenta circle indicated the guidance-commanded descent rate. Yellow pitch, roll, and yaw "flight director" needles were also displayed depending upon the experimental condition. The needles provided "fly-to" pitch, roll, and yaw attitude guidance to fly the trajectories to landing. Additional, less-critical status mode information was shown in periphery.

The ND provided a "gods-eye" view of ownship position and the landing zone. The ND was always drawn with ownship in the center, heading up, and the range scale automatically adjusted to keep the landing zone on the display. The ND transitioned from a flight-mode to a hover-mode once the ground speed was less than 38 knots. The VSD transition to a hover-mode at a ground speed less than 14 knots.

- In flight-mode, the ND depicted the landing zone by a magenta-colored hexagon symbol.

- In hover-mode (see Figure 3), the landing zone was depicted on the ND by two octagons. The octagons were sized to accurately represent the size of the desired and adequate landing performance standards. In addition, digital display of the body-axis forward and side velocities were presented with a graphical representation of this same information, indicated by a white line and cross symbol emanating from the ownship position.

- In hover-mode, a "fly-to" hover cue symbol ${ }^{20}$ was also displayed, depending upon the experimental condition. The open-loop hover cue "guidance" if followed by the EP would translate the vehicle to a hover over an indicated point without need for any closed loop guidance.

The VSD provided a side view of ownship position and the landing zone. The VSD used the same range scale as the ND. The VSD transitioned from a flight-mode and hover-mode once the ground speed was less than 13.6 knots. In flight-mode, the VSD, in addition to ownship and the landing zone, showed the terrain profile along the vehicles heading and the current vertical flight path angle. In hover-mode (see Figure 3), the flight path information was changed to show the forward and vertical speeds in graphical form and with digital read-outs. 
Radar altitude was also digitally displayed. A magenta ball was drawn to show the guidance-commanded descent rate. The landing zone depiction was colored to show the desired and adequate landing performance standards.

An Auxiliary Display (Fig. 3) was provided on the second head-down display. The Auxiliary Display showed a simulated FLIR image of the outside world from $45^{\circ}$ to $75^{\circ}$ nose-low from the DERP. This camera view supplemented the EPs visibility below the $45^{\circ}$ cut-off of the simulated window. The horizontal viewing angles subtended by the simulated FLIR camera are shown by the blue "wedge" symbol on the ND and the subtended vertical viewing angles are shown by a blue "wedge" symbol on the ND (when in the flight-mode). The simulated FLIR camera was generated from the OTW database representation where the imagery color was converted to green based on the intensity of the true color and the brightness was attenuated based on pixel distance and elevation/azimuth angle. Random noise was also inserted to approximate scintillation.

\subsection{Head-worn display}

The HWD was a prototype Rockwell Collins ProView ${ }^{\mathrm{TM}}$ SO35-MTV ${ }^{21}$ (Figure 4). This display was a transparent 800x600 pixel, monochrome green display. The field-of-view (FOV) of the display was measured to be $28.6^{\circ} \mathrm{H}$ by $21.7^{\circ} \mathrm{V}$. Coupled to this display was a prototype head tracker by Intersense, Inc based on the IS-1200 tracker. The head tracker system used a hybrid combination of optical and inertial sensors. Both the display and tracker were mounted to clear lens glasses. The head-borne weight of the combined display and tracker system was approximately $4 \mathrm{oz}$ (113 grams).

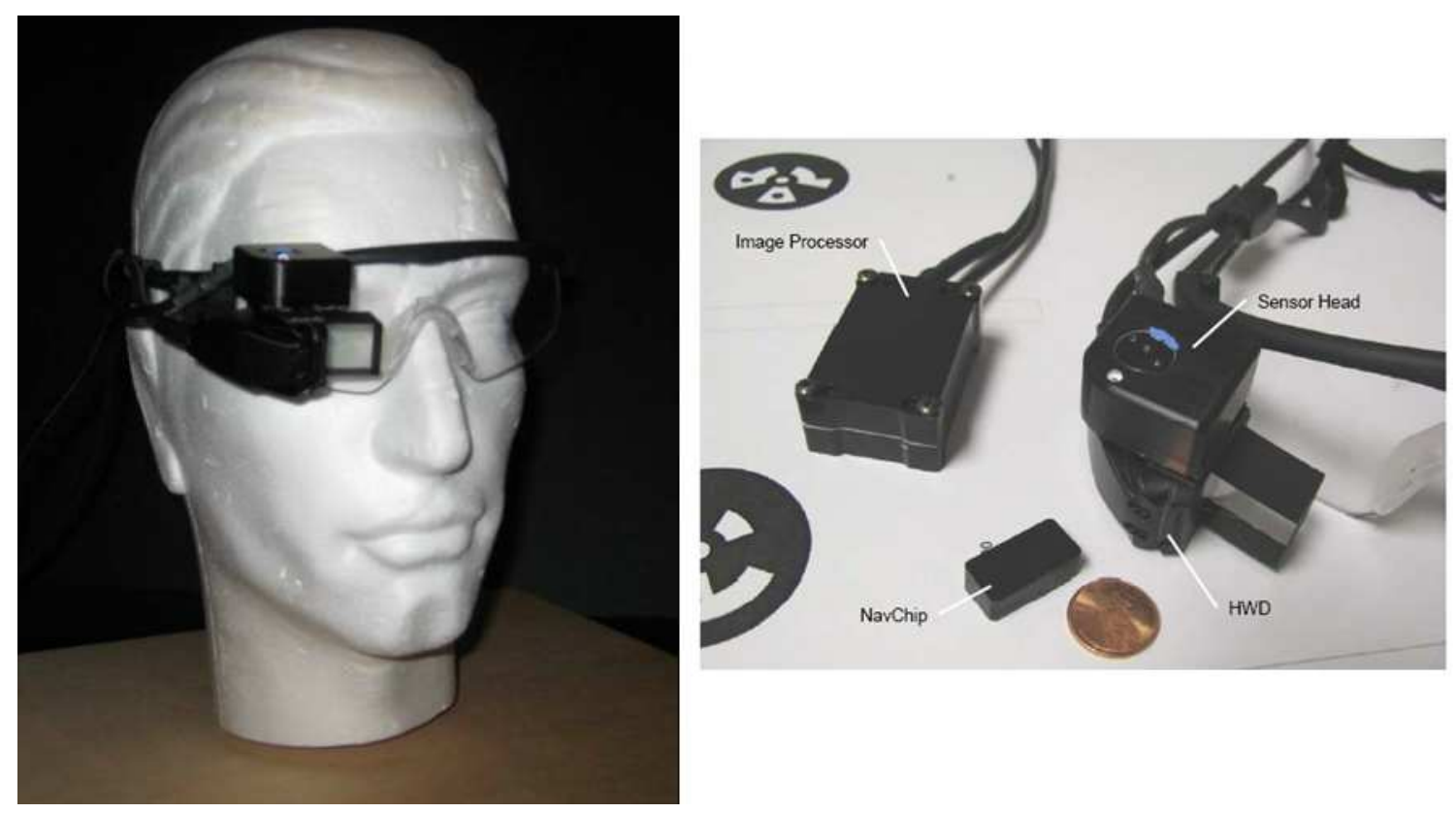

Figure 4. HWD prototype used in experiment.

At the beginning of each of the HWD data run blocks, an alignment of the HWD system was performed via a boresighting procedure to the OTW view.

The HWD imagery and symbology was mapped to the field-of-regard map as sketched in Figure 5. A "virtual" HUD was rendered using an vehicle-stabilized reference in the approximate location where an actual HUD would be. In other words, if the EP was looking forward toward the location where a HUD combiner glass would be, the virtual HUD was displayed on the HWD. The symbology was nominally an aircraft "standard" HUD information presentation (i.e., pitch ladder, tapes for altitude and ground speed) until 500 feet above the landing zone where it was replaced with non-conformal hover symbology analogous to the ND symbology.

When the EP was not looking in the area of the virtual HUD, off-boresight symbology was displayed (Figure 5) in a screen-referenced format. The off-boresight symbology consisted of an attitude indicator, digital readouts of speed, altitude and sink-rate. 
The FLIR camera image (EV sensor) was displayed on the HWD scene using a vehicle-stabilized reference. The $30^{\circ} \mathrm{FOV}$ FLIR camera was fixed to the spacecraft, pointing forward and pitched down approximately $60^{\circ}$ from the horizon.

Three conformal, terrain-referenced guidance symbols were placed on the HWD scene/imagery (i.e., either the FLIR or the SV): 1) the landing zone icon; 2) the velocity vector; and 3) a hover cue.

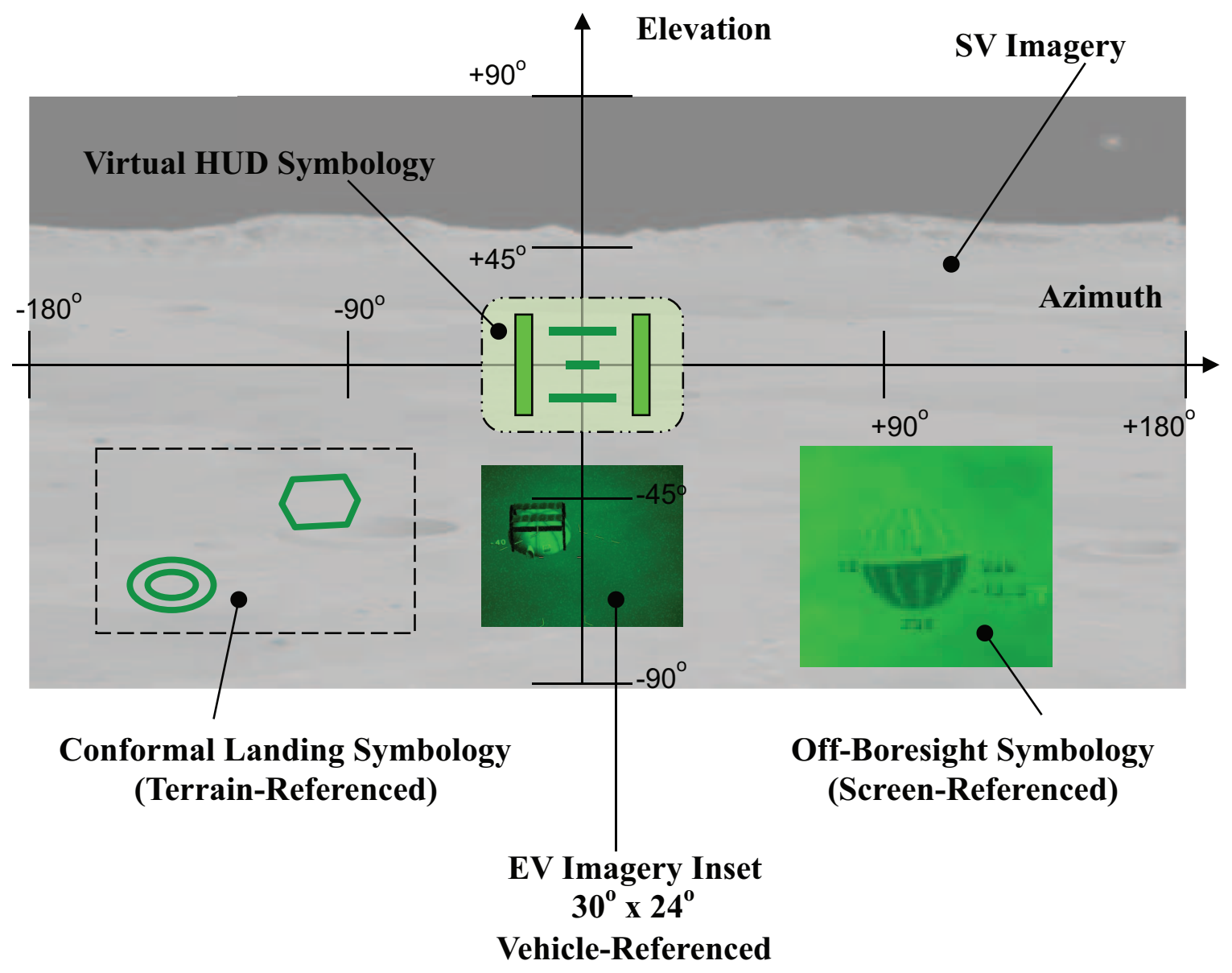

Figure 5. HWD field of regard.

Figure 6 shows sample images from the HWD. The black color in the images would actually be the transparent portions of the display. The image on the left in Figure 6 shows the virtual HUD. The image on the right shows an example of an off-boresight HWD view. In this case, the EP is looking at the landing zone (the hexagon). A portion of the FLIR camera can be seen in the left part of the display. The off-boresight attitude symbology is seen at the top of the display as well as the guidance cue and the bottom of the image (double circle). The filled green circle in the lower left of the display was a tracker integrity indicator.

\subsection{Task}

Simulated approach and landings were flown using various approach trajectories. Each run started at approximately $1000 \mathrm{ft}$ above the landing zone elevation. From the start until $500 \mathrm{ft}$ above the landing zone elevation, the approach was flown by an autopilot following a constant deceleration trajectory profile, to a landing zone directly forward of the vehicle's track. This period of auto-flight provided two benefits: 1) it allowed the pilots to prepare for the manual flight phase; and, 2) it was representative of an operational profile, where the auto-flight system would fly to a certain altitude, but the crew will take-over manually for the final landing. This procedure 


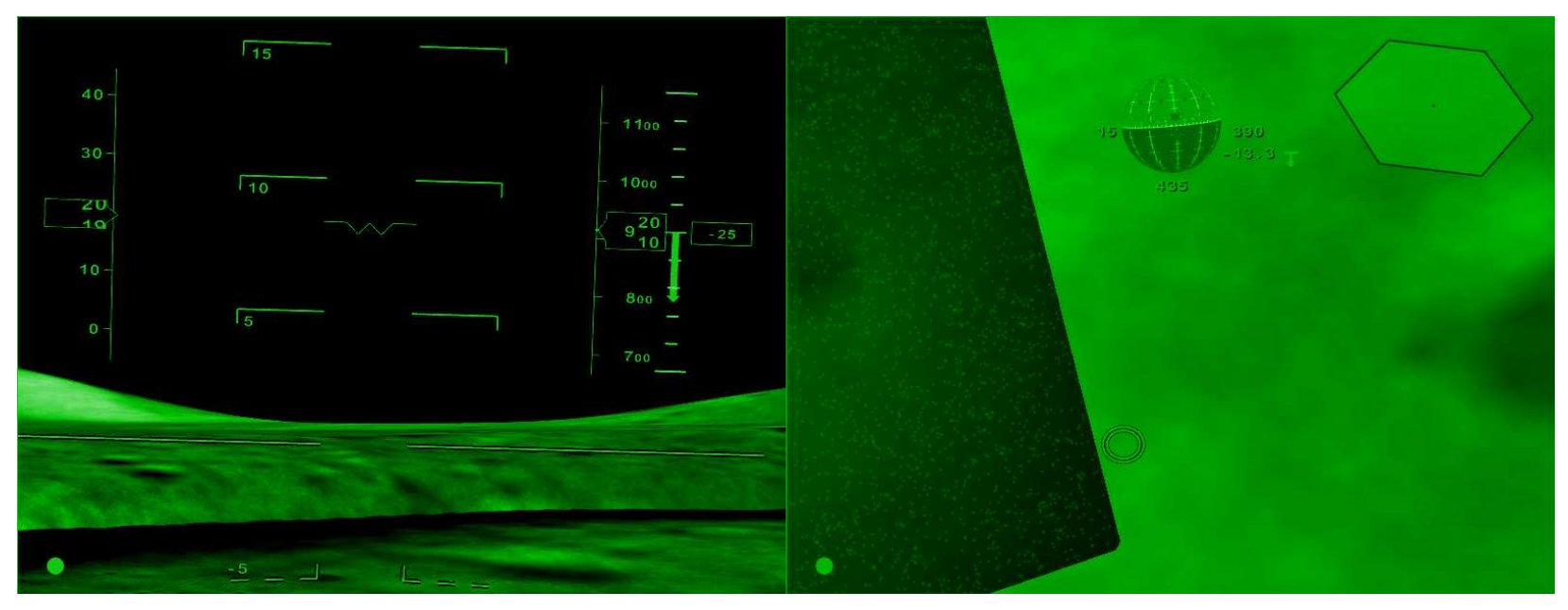

Figure 6. HWD sample images. The virtual HUD (left) and offboresight of showing landing zone (right).

was done for all Apollo flights ${ }^{22}$ and will likely occur for other crewed missions. An additional motivation is that this auto-flight period might be representative of a "re-designation" period where the crew might be evaluating real-time sensor data and navigation systems data to decide if the planned landing zone is safe and appropriate, or if a landing zone re-designation is necessary.

At $500 \mathrm{ft}$ above the touchdown zone, the auto-flight system was disconnected. This disconnect was annunciated by an audible tone over the cockpit intercom. Concurrently, the landing zone was automatically re-designated left or right by $250 \mathrm{ft}$ from its original position. The EP was then tasked to fly to and land on this re-designated landing zone. Depending upon the trajectory, the EP may have periods of time where the landing zone was viewable out the window. Attitude, hover guidance symbology, and other information was available on the head-down displays. For data runs with the HWD, conformal SV, EV, virtual HUD symbology (including hover guidance cue) and other information was also available. The $250 \mathrm{ft}$ offset landing zone mimicked the scenario where a re-designated landing zone had been executed and the pilot then had to fly to the new landing area. The $250 \mathrm{ft}$ offset was chosen to match previously conducted work. ${ }^{11,12}$ The pilot's task was to control the vehicle's trajectory, fly to a hover transition, and execute a vertical descent to land to achieve prescribed landing performance standards. The hover cue aided the pilot in the control of the vehicle's trajectory, to perform a hover transition, and execute a vertical descent to land.

\section{RESULTS}

\subsection{Quantitative}

Three factors were compared between runs with and without the HWD. An Analysis of Variance (ANOVA) was performed on each of the dependent variables: main fuel used, RCS fuel used, and range from intended landing point. No significant differences were found, $p>0.05$, for any of the 3 dependent variables.

\subsubsection{Latency measurement}

System time delays or latencies inherent to the head-worn (and helmet-mounted) displays have been shown to critically influence performance, utility, usability, and acceptability. Consistent definitions and measurement techniques as well as preliminary latency (time delay) requirements have been proposed for HWD S/EV applications. ${ }^{23}$

An in-situ latency measurement technique - the "windshield washer" test ${ }^{24}$ - was used to measure the HWD latency. This test involves a space-stabilized, boresight symbol and an outer "target box" of known dimensions located symmetrically on either side of the boresight symbol. In the presence of HWD latency, the boresight symbol cannot remain perfectly space-stabilized. 
The total system latency of the HWD system was measured to be 67 milliseconds. The total system latency measure included the time to measure the current head position, transmit the data to the computer, software time to read the tracker data and draw the elements of the HWD scene. Another latency measure was obtained by not rendering the lunar terrain (a condition the EPs did not experience). Removing the terrain from the HWD resulted in a total system latency of 33 milliseconds.

\subsection{Qualitative}

\subsubsection{Simulation sickness questionnaire}

Before and after each data block with the HWD, EPs were given a Simulation Sickness Questionnaire (SSQ). ${ }^{25}$ There were no significant effects using the HWD. Most EPs reported no SSQ symptoms. Of the 5 EPs who used the HWD, only one EP reported slight eye strain after the HWD data runs.

\subsubsection{NASA TLX}

An ANOVA was performed for the dependent variable of task load index (mental workload) from flight crew ratings on the NASA Task Load Index (NASA-TLX) scale. ${ }^{19}$ No significant differences were found, $p>0.05$, between the HDDs and the HWD.

\subsubsection{Pilot comments}

EPs commented that the HWD allowed for greater situational awareness (SA) by allowing the pilot to view flight critical information while remaining "eyes-out." However, the brightness of the HWD was controlled manually by two separate discrete button presses, located on a side panel in the crew station. It was not easy to adjust while conducting the landing. As the lunar surface became visible in the OTW scene, the green symbology would transition from being on the black background of space to a light gray background of the lunar surface. As the initial brightness setting was done on the back drop of space, the brightness level of the symbology was too low when rendered with the lunar surface background.

Video effects from system latency were distracting. Causes for system latency include the video refresh of the HWD, the latency in the head tracker measurement, and data transmission delays. During head movements, the latency in the system would cause the synthetic terrain to "lag" behind the OTW terrain. Further, a video phenomena occurs when the display graphics are not updated at the same refresh rate as the display device. This causes a blurring of the video which makes reading digital readouts and text unreadable.

EPs felt the FOV of the HWD was too small. While the FOV of the HWD is only slightly smaller than a HUD (typical HUD FOV is $30^{\circ}$ by $24^{\circ}$ ), the EPs felt they were viewing the world through a "soda straw."

EPs commented that the hover cue that was projected on the lunar surface of the HWD was too sensitive. The hover cue was tuned to work best on the non-conformal ND and was not tuned for the HWD.

The head tracker used an optical camera to view known target locations on the LFD cockpit ceiling to correct inertial drift of the tracker. However, the tracker was very sensitive to uneven lighting of the targets. The end result of the tracker losing the ceiling targets then reacquiring them would cause an abrupt jump in the conformal symbology, most noticeable in the synthetic terrain alignment.

\subsection{Discussion of results}

In general, EPs thought the HWD had great potential but was not optimized for this lunar landing task. The performance data shows there were no significant performance differences when using the HWD in conjunction with the HDDs. Also, there were no significant workload or simulator sickness effects with the HWD. Regarding situational awareness, the data is anecdotal. Some pilots felt the HWD provided greater SA because the HWD allowed for an eyes-out view while still being able to perform the task. EPs preferred this eyes-out view as it provided "truth" data as to what the actual situation is, rather than relying on a computed navigation solution which can be subject to errors. These comments were from EPs who were familiar with and frequently flew night vision systems which are monocular and monochrome green; therefore they were used to and familiar with having a monocular display over one eye while flying. 
However, those EPs who had not flown such systems were distracted by the HWD at times. The artifacts associated with a head tracked display, such as blurry text and numbers, caused EPs to abandon using the HWD and rely solely on the HDDs. Thus, EPs would try keep their heads still in order to maximize readability of the display or rely on the HDD.

The presence of SV and EV imagery on the HWD did not aid the EPs in conducting the landing task in this experiment. The primary task of this experiment was to safely land the spacecraft which was challenging. The nature of lunar landing spacecraft requires EPs to constantly monitor the attitude and sink rate in order to land in the designated landing zone (a $15 \mathrm{ft}$ radial area in this experiment). Failure to maintain the proper attitude would result in a state that would be unrecoverable for a safe landing. As a result, EPs tended to rely on the hover cue guidance to provide the proper attitude and setup for the landing. As EPs were task-saturated with maintaining the proper attitude and a safe sink rate, the task of monitoring the S/EV imagery might be best offloaded to a non-flying crew member.

Further, as EPs were already eyes-out when using the hover cue on the HWD, the SV terrain reduced the contrast of the symbology. Also, when looking at the head-down displays, the S/EV imagery could obscure symbology. This suggests that there should be "black-out" zones where if the pilots are looking at certain areas, the S/EV imagery would be removed.

The FLIR image was sized to represent a typical FOV of an aviation FLIR camera. The simulated FLIR camera was placed to provide additional look-down in the OTW area that is obscured by the spacecraft deck (see Fig. 1). However, given the approach angles, speeds on approach and the FOV of the FLIR camera, the landing zone was rarely in the FLIR image. It was suggested to move the FLIR camera to point directly below the spacecraft; however this would be physically impossible (due to the descent engine nozzle occluding this view) and it is unknown if the thermal signature of the descent engine would over power the camera. A second suggestion was made of mounting a FLIR camera to each of the lunar lander's pads. This would provide assurance the landing zone was clear of hazards.

Pilot comments from previous experiments with HWDs expressed a desire for a small, light-weight display that can be comfortably worn. ${ }^{2}$ The HWD system used in this experiment was light-weight especially when compared to previous systems. One of the trade-offs to having a small system is the FOV of the HWD is smaller than a HUD. Even though the system has an unlimited field of regard, the small FOV made it difficult to target interesting areas. All EPs agreed that a HWD with a larger FOV would be desirable; however, EPs were not asked to quantify how much larger the FOV should be.

Binocular rivalry effects were not observed with the HWD system used in this experiment. Binocular rivalry is a well documented phenomena where two vastly different images are presented to each eye. When two disparate images are presented to each eye, the brain involuntarily suppresses one of the images. Even though a monocular HWD system was used in this experiment, binocular rivalry was not so much an issue as the tranparent HWD allows for similar images to the eyes. ${ }^{26}$

Increasing the FOV would come at a cost of weight and reduced resolution. ${ }^{27}$ For targeting tasks, research has shown that targeting improves as the FOV increases up to $40^{\circ} .{ }^{26}$ After $40^{\circ}$, performance improvements where not significant. ${ }^{26}$

From the latency measurements, it can be seen that rendering the terrain doubles the latency from $33 \mathrm{~ms}$ (without terrain) to $67 \mathrm{~ms}$. Previous studies have shown that it is desirable to have the HWD system latency no more than $20 \mathrm{~ms}^{24}$ Reducing the latency to $20 \mathrm{~ms}$ would result in more readable text and reduce blurring video artifacts. However, the minimal latency of a display refreshed at $60 \mathrm{~Hz}$ is $17 \mathrm{~ms}$ without processing, indicating a signficant challenge for video processing, etc. Therefore, improving the terrain rendering efficiency can greatly reduce the overall system latency. This can be achieved by improving the software rendering algorithms or waiting for more powerful graphics cards. 


\section{CONCLUSIONS}

A head-worn display concept, coupled with a head tracker, was used to evaluate full-field-of-regard S/EV concepts for a lunar lander simulation. A fixed-based simulation experiment evaluated an initial application of using a head-worn display to improve situational awareness for lunar landing operations. The simulation experiment was conducted at a simulated Apollo 15 landing site using test pilots and astronauts and various display concepts. In general, EPs thought the HWD has great potential but was not completely optimized for this lunar landing task. There were no workload, simulation sickness or performance effects from using the head tracked HWD system. EPs commented that the HWD allowed for greater SA by allowing the pilot to view flight critical information while remaining "eyes-out." While the HWD small size has the advantage of being light, thus comfortable, EPs felt the FOV was too small.

\section{FURTHER RESEARCH}

The encumbrance of the HWD was significantly less that previous NASA studies; however work must continue toward reducing the system latency. These results were obtained using a ground simulation facility with no motion effects. These results and others must be validated during actual operations, focusing on daylight readability (particularly for evaluating the utility and capabilities for color), disorientation and illusion issues (i.e., simulator sickness), and augmented reality requirements.

\section{REFERENCES}

[1] Bailey, R., Jackson, E., Arthur, J., and Barnes, J., "Control and display interaction for lunar landing vehicle handling qualities," submitted to AIAA (2010).

[2] Arthur III, J. J., Prinzel III, L., Bailey, R., Shelton, K., Williams, S., Kramer, L., and Norman, R., "Headworn display concepts for surface operations for commerical aircraft," Tech. Rep. NASA/TP-2008-215321, NASA Langley Research Center, Hampton, VA (June 2008).

[3] Stengel, R., "Manual attitude control of the lunar module," Journal Of Spacecraft And Rockets 7, 941-948 (1970).

[4] Slayton, D., "Apollo: Past, present and future," in [13th Symposium Proceedings], The Society of Experimental Test Pilots (September 1969).

[5] Hackler, C., Brickel, J., Smith, H., and Cheatham, D., "Lunar module pilot control considerations," Tech. Rep. NASA TN D-4131, NASA (February 1968).

[6] Williams, S., Arthur III, J., Shelton, K., Prinzel III, L., and Norman, R., "Synthetic vision for lunar and planetary landing vehicles," in [Enhanced and Synthetic Vision 2008], Guell, J. and Uijt de Haag, M., eds., SPIE (2008).

[7] Bailey, R., Kramer, L., and Prinzel III, L., "Crew and display concepts evaluation for synthetic / enhanced vision systems," in [Enhanced and Synthetic Vision Proceedings of SPIE], Verly, J. G., ed., 6226-25, SPIE (April 2006).

[8] Arthur III, J., Kramer, L., Bailey, R., and Prinzel III, L., "Flight test comparison between enhanced vision (FLIR) and synthetic vision systems," in [Enhanced and Synthetic Vision 2005], Verly, J. G., ed., 5802, SPIE (April 2005).

[9] Harris, D. and Muir, H., eds., [Contemporary Issues in Human Factors and Aviation Safety], Ashgate (2005). http://www.ashgate.com/default.aspx?page=637\&calcTitle=1\&isbn=9780754645498\&amp;lang=cy-GB.

[10] Williamson, M., [Spacecraft Technology: The Early Years], The Institution of Electrical Engineers (2006).

[11] Mueller, E., Bilimoria, K., and Frost, C., "Effects of control power and inceptor sensitivity on lunar lander handling qualities," in [SPACE 2009 Conference and Exposition], AIAA (September 2009). AIAA Paper 2009-6407.

[12] Bilimoria, K., "Effects of control power and guidance cues on lunar lander handling qualities," in [SPACE 2008 Conference and Exposition], AIAA (September 2008). AIAA Paper 2008-7799.

[13] Cohen, M., "From Apollo LM to Altair: Design, environments, infrastructure, missions, and operations," in [SPACE 2009 Conference and Exposition], AIAA (September 2009). AIAA Paper 2009-6404. 
[14] Duda, K., Johnson, M., and Fill, T., "Design and analysis of lunar lander manual control modes," in [2009 IEEE Aerospace Conference], IEEE (March 2009).

[15] Mindell, D., [Digital Apollo: Human and Machine in Spaceflight], The MIT Press (2008).

[16] Michael M., M., "Architecting a simulation framework for model rehosting," in [AIAA Modeling and Simulation Technologies Conference], AIAA (August 2004).

[17] Isbell, C., Eliason, E., Becker, T., Lee, E., and Robinson, M., "The Clementine mission: An archive of a digital image model of the moon," in [28th Annual Lunar and Planetary Science Conference], 623-624, Lunar and Planetary Institute (March 1997).

[18] Cooper, G. and Harper Jr., R., "Use of pilot rating in the evaluation of aircraft handling qualities," Tech. Rep. NASA TN D-5153, NASA (April 1969).

[19] Hart, S. G. and Staveland, L. E., "Development of a multi-dimensional workload rating scale: Results of empirical and theoretical research," in [Human mental workload], Hancock, P. A. and Meshkati, N., eds., 139-183, Elsevier, Amsterdam, The Netherlands (1988).

[20] Schroeder, J. and Merrick, V., "Control and display combinations for blind vertical landings," Journal of Guidance, Control, and Dynamics 15, 751-760 (May-June 1992).

[21] "Rockwell Collins ProView SO35-MTV," http://rockwellcollinsaviation.org/content/pdf/pdf_11503.pdf.

[22] Major, L., Brady, T., and Paschall II, S., "Apollo looking forward: Crew task challenges," in [2009 IEEE Aerospace Conference], IEEE (March 2009).

[23] Bailey, R. E., Arthur III, J. J., Prinzel III, L. J., and Kramer, L. J., "Evaluation of head-worn display concepts for commercial aircraft taxi operations," in [Head-and Helmet-Mounted Displays XII: Design and Applications], Rash, C. E. and Reese, C. E., eds., 6557, SPIE (April 2007).

[24] Bailey, R. E., Arthur III, J. J., and Williams, S., "Latency requirements for head-worn display s/evs applications," in [Enhanced and Synthetic Vision 2004], Verly, J. G., ed., 5424, 98-109, SPIE (April 2004).

[25] Kennedy, R. S., Lane, N. E., Berbaum, K. S., and Lilienthal, M. G., "Simulator sickness questionnaire: An enhanced method for quantifying simulator sickness," International Journal of Aviation Psychology , 203-220 (1993).

[26] Patterson, R., Winterbottom, M., and Pierce, B., "Perceptual issues in the use of Head-Mounted visual displays," Tech. Rep. AFRL-HE-AZ-TP-2007-03, Air Force Research Laboratory, Mesa, AZ (2006).

[27] Cupero, F., Valimont, B., Wise, J., Best, C., and Mers, B. D., "Head worn display system for equivalent visual operations," Tech. Rep. NASA/CR-2009-215781, Hampton, VA (July 2009). 